\title{
Embedded Monitoring of QoS Parameters in IP-Based Virtual Private Networks
}

\author{
T. Lindh \\ The Department of Microelectronics and Information Technology at KTH \\ and Telia ProSoft \\ (thomas@haninge.kth.se)
}

\begin{abstract}
In this paper we propose a framework for performance monitoring that is based on embedded monitoring, where OAM packets are inserted between blocks of packets with user data.
\end{abstract}

\subsection{A Framework for Embedded Monitoring}

We briefly describe a framework for embedded performance monitoring of QoS parameters in IP networks, especially virtual private networks. The proposed architecture has two main characteristics: first, it uses an in-service method in which parameters in the actual user traffic are measured by means of dedicated monitoring packets; and secondly, the monitoring functions form an integrated part of the ordinary network elements.

Measurement activities have to be subordinated to the operator's policy and objective for performance management, related to service level agreements and operational needs. There is no meaning to carry out extensive performance monitoring for its own sake. This study is focused on IP-based virtual private networks since they represent a case where more elaborated performance monitoring clearly is motivated. The assumed topology with a core network surrounded by edge routers is described in section 2. There are some related work reported in a previous paper by [Beigi] and in the results from the EURESCOM project QUASIMODO [Jormakka].

\subsection{Requirements for Connectionless Networks}

Performance monitoring in networks based on connectionless protocols such as IP is not trivial. In connection-oriented protocols, e.g. ATM, every packet or cell belongs to a logical connection with a predetermined path. However, an IP datagram carries only the network addresses of the source and the destination, and there is no guarantee that all packets in a session traverse the same path from sender to receiver and arrive in the original order. Furthermore, IP permits variable packet size, which has to be considered when estimating throughput as well as the packet loss ratio.

\subsection{Embedded OAM Packets}

The purpose of embedded monitoring is to measure relevant network performance parameters based on the actual user traffic. These dedicated monitoring packets, 
OAM (operation, administration and maintenance) packets, are inserted between blocks of ordinary data packets as shown in Figure 1. The sending node generates monitoring packets that convey monitoring information between every $\mathrm{N}$ user packets on average. The receiving node detects the monitoring packets, adds information and returns them to the originating node. Processing, storage and analysis may be carried out by dedicated servers for the entire network. Synchronisation of the clocks in the monitoring nodes, for measuring one-way delay, can be carried out using for example the Global Positioning System and the Network Time Protocol. It is possible to obtain the following results using the method described above:

- The number of lost packets between sending and receiving nodes and the packet loss ratio during the measurement period.

- The length of the loss-free periods and the loss periods expressed in terms of the number of consecutive OAM blocks that contain lost packets and the number of OAM blocks that are loss-free.

- Samples of the transfer delay, and delay variations, between sending and receiving nodes.

- An estimate of the utilised capacity (throughput) between each pair of sendingreceiving edge nodes. This requires that the mean packet length can be obtained.

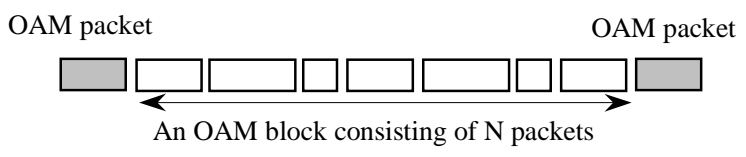

Figure 1: Two OAM packets enclose an OAM block that consists of $\mathrm{N}$ user packets on the average.

\subsection{Monitoring of Traffic in Virtual Private Networks}

The goal is to measure and estimate losses, delays and throughput for IP traffic in virtual private networks between provider edge nodes or customer edge nodes, based on the general topology in Figure 2. Virtual private networks can be implemented in different ways. In router-based networks so called tunnels are created by means of overlay point-to-point connections, using for example generic route encapsulation or IPSec. Multi Protocol Label Switching promises to provide a more flexible and scalable framework for VPNs based on cell switches or a mixed environment with routers and switches.

As mentioned previously, IP's connectionless nature is a major problem in designing monitoring systems. To use OAM packets in IP networks requires that an entrymonitoring (sending) node can find out, based solely on the destination IP address in the packet header, which of the exit-monitoring (receiving) nodes the packet will traverse. Similarly, a receiving node has to determine from which sending node an arriving packet was sent. In addition, the receiving node must be able to compute the average packet size for the OAM blocks, in order to estimate throughput and utilisation. The solution for a general IP network requires efficient look-up functions in order to determine the correct exit (and entry) monitoring nodes. However, it turns out 
that in several important cases the complexity of the problem is considerably reduced. In virtual private networks, implemented by means of point-to-point tunnels, the address of the exit node is carried in an additional IP header. Therefore it is feasible to implement a look-up function in the monitoring node that returns the correct exitmonitoring node given an IP destination address. In more general cases a similar procedure might require more complex mapping functions.

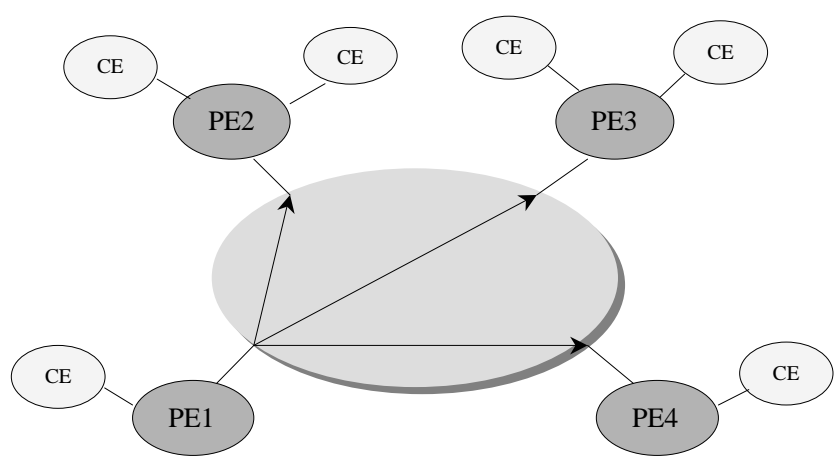

Figure 2: A topology for virtual private networks. The provider edge node 1 (PE1) acts an entry-monitoring node for the outgoing traffic and the receiving nodes act as exit-monitoring nodes for the incoming traffic. Corresponding monitoring functions may also be implemented in customer edge nodes (CE) as well as in individual hosts.

\subsection{Functions in Monitoring Nodes}

The monitoring functions in the nodes can be configured through a policy-server, e.g. in accordance with the Directory Enabled Network (DEN) architecture. These functions are activated if specific sets of conditions that express the operator's monitoring policy are fulfilled, for example that specific service classes should be monitored during certain time periods.

When acting as an entry-monitoring node, a router should perform the following monitoring functions for each VPN (and correspondingly as an exit-monitoring node). It should maintain separate counters for each exit-monitoring node. These counters keep track of the number of packets that are sent to each of the receiving nodes. Since the IP addresses of the tunnel endpoints as well as the connected network addresses are known, the translation from a destination IP address in the packet header to the corresponding exit-monitoring node is feasible. The OAM packets are generated by the ingress node and inserted periodically into the user traffic between blocks user packets with the destination address pointing to the exit-monitoring node. The source address is set to the address of the entry-monitoring node itself. The payload of the OAM packet contains the following information:

- A sequence number attached to each OAM packet.

- The entry-monitoring node inserts the total number of packets sent to the exit node (cumulative) and a timestamp that shows when the packet is transmitted.

- The exit-monitoring node inserts the total number of received packets and a timestamp when the packet arrives. 
Since the generation of OAM packets may compete with the forwarding of data packets, the distance between monitoring packets will depend on the current traffic load. An increased block length during periods of heavy traffic can be adjusted to a desired average length when the router is less loaded. An advantage of a varying block size might be that correlation with periodic traffic patterns could be avoided. For an optimal choice of the distance between OAM packets the sample rate of the delay and jitter measurements as well as the granularity of the loss information also have to be considered, related to the applicable levels of the service agreements with customers.

\subsection{Results}

The difference between the number of packets sent in OAM packet $n$ and OAM packet $n-1$ gives the number of packets sent in an OAM block, and correspondingly for the number of received packets. Thus, subtracting these two results yields the number of packets that has been lost in the OAM block. Since losses in packet networks tend appear in bursts, mainly due to overflow in buffers, there is a need to define a metric that also covers these characteristics of the loss process. The length of the loss period and the loss-free period can be measured in terms of the number of consecutive OAM blocks with and without losses. Since the number of packets transmitted (and received) and timestamps for every OAM packet are recorded at every edge node, it is possible to obtain an estimate of the throughput between the edge nodes.

Since the packet size is not fixed, it is desirable to express the throughput in terms of bits per second and the packet loss based on bytes or bits, and not merely on the number of packets. This can be obtained if the monitoring nodes collect samples of the packet length from the IP header and computes an estimate of the mean packet length and its distribution.

\subsection{Summary and Ongoing Work}

In this paper we have briefly presented an infrastructure for monitoring of performance parameters in IP networks, with a special focus on virtual private networks. A more detailed description and discussion of the method and ongoing implementation can be found in [Lindh].

\section{References}

[Beigi] Beige M., Jennings R., Verma D.: “Low Overhead Continuous Monitoring of IP Network Performance", Proceedings of Symposium on Performance Evaluation of Computer and Telecommunication Systems, SPECTS'99, Chicago, July 1999.

[Jormakka] Jormakka J., Heikkinen K.: "QoS/GOS Parameter Definitions and Measurement in IP/ATM Networks", Proceedings of International Workshop QofIS 2000, Germany, September 2000.

[Lindh] Lindh T.: "An Architecture for Embedded Monitoring of QoS Parameters in IP Based Virtual Private Networks", Passive and Active Measurements 2001, Amsterdam, 23-24 April 2001. 